\title{
Bioinformatics analysis identifies miR-221 as a core regulator in hepatocellular carcinoma and its silencing suppresses tumor properties
}

\author{
XING-XING HE ${ }^{1 *}$, AN-YUAN GUO ${ }^{2 *}$, CHUAN-RUI XU ${ }^{3}$, YING CHANG $^{1}$, GUANG-YA XIANG ${ }^{3}$, \\ JING GONG $^{2}$, ZI-LI DAN ${ }^{1}$, DE-AN TIAN ${ }^{1}$, JIA-ZHI LIAO ${ }^{1}$ and JU-SHENG LIN ${ }^{1}$ \\ ${ }^{1}$ Institute of Liver Diseases, Tongji Hospital, Tongji Medical College, Huazhong University of Science and Technology, \\ Wuhan, Hubei 430030; ${ }^{2}$ Hubei Bioinformatics and Molecular Imaging Key Laboratory, Department of \\ Biomedical Engineering, College of Life Science and Technology, Huazhong University of Science \\ and Technology, Wuhan, Hubei 430074; ${ }^{3}$ School of Pharmacy, Tongji Medical College, \\ Huazhong University of Science and Technology, Wuhan, Hubei 430030, P.R. China
}

Received March 21, 2014; Accepted June 17, 2014

DOI: $10.3892 /$ or.2014.3306

\begin{abstract}
Hepatocellular carcinoma (HCC) is a worldwide malignancy; however, there is a lack of effective targeted therapies. We and others have found that miR-221 is one of the most consistently overexpressed miRNAs in liver cancer. However, the roles of miR-221 in hepatocellular carcinogenesis are still not fully elucidated. In the present study, we used bioinformatics tools, gain- and loss-of-function methods to determine the roles of miR-221 in HCC. Bioinformatics analysis showed that miR-221 is a core miRNA which targets a large number of HCC-related genes and has formed many feed-forward regulatory loops combining transcription factors (TFs) to regulate HCC-related genes. Inhibition of miR-221 in liver cancer cells decreased cell proliferation, clonogenicity, migration/invasion and also induced G1 arrest and apoptosis. In addition, we demonstrated that miR-221 bound directly to the 3'-untranslated region of BMF, BBC3 and ANGPTL2, and inhibited the expression of BMF, BBC3 and ANGPTL2. In a mouse model, lentivirus-mediated miR-221 silencing could significantly suppress the growth of hepatoma xenografts in nude mice. In conclusion, we showed that miR-221 is a critical modulator in the HCC signaling pathway, and miR-221 silencing inhibits liver cancer malignant properties in vitro and in vivo, which may benefit the treatment for patients with unresectable HCC.
\end{abstract}

Correspondence to: Dr Jia-Zhi Liao or Dr Ju-Sheng Lin, Institute of Liver Diseases, Tongji Hospital, Tongji Medical College, Huazhong University of Science and Technology, 1095 Jie-Fang Avenue, Wuhan, Hubei 430030, P.R. China

E-mail: liaojz2013@126.com

E-mail: jslin@tjh.tjmu.edu.cn

*Contributed equally

Key words: bioinformatics analysis, liver cancer, ANGPTL2, miR-221

\section{Introduction}

Hepatocellular carcinoma (HCC) is the fifth most common cancer worldwide and the third most common cause of cancerrelated mortality, with $>600,000$ deaths/year (1). The heavy burden of HCC has generated extensive studies of molecular mechanisms underlying this disease in the hope of elucidating its development and determining better methods for treatment.

MicroRNAs (miRNAs) are evolutionarily conserved, small non-coding RNAs that are considered to play fundamental roles in various biological processes through regulation of gene expression at the level of post-transcription. Several studies have reported some important aberrant miRNA expression profiles in HCC and found multiple specific dysregulated miRNAs associated with the development of liver cancer through regulating oncogenes or tumor suppressors (2-8). Transcription factors (TFs) are essential for the regulation of gene expression by binding to specific DNA sequences on the promoter of target genes. Many studies have proved that TFs are key regulators in HCC, in particular, the protooncogenes c-Myc, c-fos and c-jun are frequently overexpressed in $\mathrm{HCC}$ and play critical roles in modulating cellular growth, differentiation and apoptosis by regulating a number of genes (9-12). Both the TFs and miRNAs are key regulators of gene expression, and they may mutually regulate each other to form feedback loops, or they regulate the same target gene to form a feed-forward loop (FFL) (13). For example, the cyclin D1 and miRNA-17/20 feedback loop in breast cancer and TP53/miR106b/E2F FFL in cell proliferation have been experimentally verified $(14,15)$. However, the combined regulation of miRNAs and TFs in HCC remains elusive.

Previously, we identified a specific aberrant miRNA expression profiling in HCC by comparison of miRNA expression profiles in cancerous hepatocytes with normal primary human hepatocytes and found that miR-221 was the most overexpressed miRNA in HCC (16). Several other studies also demonstrated that miR-221 was one of the most upregulated miRNAs in liver cancer cell lines and tissues 
$(6,8,17)$. Consistently, upregulation of miR-221 in glioblastoma, lung, liver, stomach, colon, pancreatic, kidney, bladder, prostate and thyroid cancer strengthened its importance in tumorigenesis $(8,17-24)$. Recently, Callegari et al developed a liver-specific overexpression of miR-221 transgenic mouse model and found that these miR-221 transgenic animals exhibited a strong predisposition to the development of liver tumors (25). miR-221 can promote cell proliferation and increase the cell number in S-phase by suppressing p27 (18), p57 (26) and PTEN (17); inhibit cell apoptosis by modulating BMF (27); accelerate angiogenesis by regulating TIMP3 (17); and regulate DNA damage and repair by targeting DDIT4 (8). These studies strongly suggested an important role of miR-221 upregulation in hepatocarcinogenesis; however, little is known regarding its upstream regulatory mechanisms and there is a lack of a comprehensive understanding of miR-221 in the HCC signaling pathway.

In the present study, bioinformatics analysis was used to reveal dysregulated miRNAs in modulating the signaling network of HCC. We focused on upregulated miR-221 in HCC, and studied the functions of miR-221 silencing in liver cancer in vitro and in vivo. In particular, the therapeutic value of stable miR-221 silencing by lentivirus-mediated-anti-miR-221 was evaluated in nude mice bearing hepatoma xenografts.

\section{Materials and methods}

Bioinformatics analysis. We previously identified a specific miRNA expression profiling in liver cancer and the microarray data were deposited in NCBI's Gene Expression Omnibus (GEO) public database (http://www.ncbi.nlm.nih. gov/geo/, GEO accession no. GSE20077) (16). Here, the most differentially expressed miRNAs were used for further bioinformatics analysis. We obtained the candidate miRNA targets using the combinatorial utilization of two different databases [TargetScan (28) and miRanda (29)] as our previous study (13) and chose the overlapped predicted targets based on evolutionary conservation among mammalian. Then, we merged the overlap targets with the experimentally reported miRNA targets from TarBase and miR2Disease resource $(30,31)$. Finally, we chose the candidate genes involved in HCC from the predicted targets as our interested miRNA targets. To characterize the FFLs among miRNA, TF and HCC genes, we parsed the ENCODE ChIP-Seq data from the UCSC genome browser database (http://genome.ucsc.edu/) to obtained the TF targets (32). For those $>100$ TFs in ENCODE data, we identified 29 TFs, which were reported to be related with HCC through literature review. Using the strategy we previously worked, we obtained the FFLs among miRNA, TF and HCC genes (13). The miRNA regulatory network was constructed by merging the FFLs and miRNA target pairs through our inner Perl scripts. The presented network images were drawn using the Cytoscape software (version 2.6.1) (33).

Cell lines, miR-221 precursor/inhibitor and cell transfection. The hepatoma cell lines SK-HEP-1, HepG2, SMMC-7721 and cervical cancer cell line HeLa were cultured and maintained as previously described (16). Stability-enhanced miR-221 precursor (pre-miR-221), miR-221 inhibitor (anti-miR-221) and their matched negative controls (pre-miR-NC and anti-miR-
Table I. Primers used in SYBR-Green qRT-PCR.

\begin{tabular}{ll}
\hline Gene name & \multicolumn{1}{c}{ Primers } \\
\hline BMF & F: 5'-CCAGCCTCCCAGCTAAAG-3' \\
BMF & R: 5'-CCTGGGGATGAACAAAATG-3' \\
BBC3 & F: 5'-TGGGACTCCTGCCCTTAC-3' \\
BBC3 & R: 5'-GGCTGGGAGTCCAGTATG-3' \\
ANGPTL2 & F: 5'-GTTTGGTACTGTCCATGTCTG-3' \\
ANGPTL2 & R:5'-GCAGATTCGTGTCATTACAAG-3'
\end{tabular}

F, forward; R, reverse.

NC) were from Ambion (Austin, TX, USA). Cell transfections were performed using Lipofectamine ${ }^{\mathrm{TM}} 2000$ (Invitrogen, Carlsbad, CA, USA) according to the manufacturer's instructions.

RNA and protein extraction. The mirVana ${ }^{\mathrm{TM}}$ PARIS $^{\mathrm{TM}}$ kit (Ambion) was applied to isolate total RNA and protein from the same experimental samples according to the manufacturer's instructions.

TaqMan $q R T-P C R$. The expression of mature miRNAs was assayed using the TaqMan MicroRNA Assays specific for hsa-miR-221 and RUN6B (both from Applied Biosystems, Foster City, CA, USA) according to the manufacturer's instructions.

Cell proliferation, cell cycle/apoptosis, cell migration/invasion and clonogenicity assay. These assays were detected as we previously described (16).

SYBR-Green $q R T-P C R$. SK-HEP-1 cells were harvested for RNA extraction $48 \mathrm{~h}$ after transfection. The First Strand cDNA Synthesis kit (Fermentas, Burlington, VT, USA) was applied to synthesize the first strand cDNAs from $1 \mu \mathrm{g}$ of total tested RNA. A $20 \mu 1$ PCR reaction/well in 384-well reaction plates was prepared by using the Platinum ${ }^{\circledR}$ SYBR-Green qPCR SuperMix UDG reagent (Invitrogen). The PCR reaction was carried out on Applied Biosystems 7900HT Fast Real-Time PCR System. GAPDH RNA was used as a control. The primer sequences are listed in Table I.

Luciferase assay. To verify the targets of miR-221, the pMIR-REPORT ${ }^{\mathrm{TM}}$ system (Applied Biosystems) was applied. Briefly, 55-mer double-stranded oligonucleotides containing the predicted miR-221 binding sites were synthesized with the single-stranded overhangs of the restriction sites SpeI and HindIII, and inserted into the multiple cloning site of the pMIR-REPORT ${ }^{\mathrm{TM}}$ luciferase vector to establish the pLUCtargets i.e. pLUC-BMF, pLUC-BBC3 and pLUC-ANGPTL2 vectors. As a control, the pLUC-muttargets, i.e. pLUCmutBMF, pLUC-mutBBC3, pLUC-mutANGPTL2 plasmids, were also prepared by the same method, except for the synthesized oligonucleotides containing corresponding mutated nucleotides in the seed-match sequence. The mutated 
Table II. The sequences of 55-mer double-stranded oligonucleotides containing the predicted miRNA binding sites.

\begin{tabular}{ll}
\hline Targeted gene & \multicolumn{1}{c}{ Sequence } \\
\hline BMF & F: 5'-CTAGTCAGGGGCTATCGAGGAGACCCAGTGAGAATGTAGCATTTTGTTCATCCCA-3' \\
& R: 5'-AGCTTGGGATGAACAAAATGCTACATTCTCACTGGGTCTCCTCGATAGCCCCTG $A-3^{\prime}$ \\
Mutated BMF & F: 5'-CTAGTCAGGGGCTATCGAGGAGACCCAGTGAGATGTATCGATTTGTTCATCCCA-3' \\
& R: 5'-AGCTTGGGATGAACAAAATCGATACATCTCACTGGGTCTCCTCGATAGCCCCTGA-3' \\
BBC3 & F: 5'-CTAGTGGCCAGCGCGGGGGACTTTCTCTGCACCATGTAGCATACTGGACTCCCA $A-3^{\prime}$ \\
& R:5'-AGCTTTGGGAGTCCAGTATGCTACATGGTGCAGAGAAAGTCCCCGCGCTGGCCA-3' \\
Mutated BBC3 & F: 5'-CTAGTGGCCAGCGCGGGGGACTTTCTCTGCACCTGTATCGATACTGGACTCCCA $A-3^{\prime}$ \\
& R:5'-AGCTTTGGGAGTCCAGTATCGATACAGGTGCAGAGAAAGTCCCCGCGCTGGCCA-3' \\
ANGPTL2 & F: 5'-CTAGTTACCTCAGCATTTCTCACAAAGTGTACCATGTAGCATGTT TTGTGTATA $A-3^{\prime}$ \\
& R: 5'-AGCTTTATACACAAAACATGCTACATGGTACACTTTGTGAGAAATGCTGAGGTAA-3' \\
Mutated ANGPTL2 & F: 5'-CTAGTTACCTCAGCATTTCTCACAAAGTGTACCTGTATCGATGTTTTGTGTATA $A-3^{\prime}$ \\
& R: 5'-AGCTTTATACACAAAACATCGATACAGGTACACTTTGTGAGAAATGCTGAGGTA $A-3^{\prime}$ \\
\hline
\end{tabular}

The sequences in bold refer to predicted pairing nucleotide with the seeding sequences of corresponding miRNAs. Italics refer to overhangs of restriction enzyme sites. F, forward; $R$, reverse.

scrambling sequences were prepared by the online tool on the web site https://www.genscript.com/ssl-bin/app/scramble. Synthesized sequences are listed in Table II. In 96-well plates, SK-HEP-1 cells were cotransfected with $0.1 \mu \mathrm{g}$ of each pLUCtarget or pLUC-muttarget, $0.01 \mu \mathrm{g}$ of pMIR-REPORT ${ }^{\mathrm{TM}} \beta$-gal plasmid served as an internal transfection efficiency control, and pre-miR-221 or pre-miR-NC with a $50 \mathrm{nM}$ final concentration. At $24 \mathrm{~h}$ post-transfection, luciferase and $\beta$-galactosidase activities were measured using the Dual-Light Assay System (Applied Biosystems) according to the manufacturer's instructions.

Western blot analysis. SK-HEP-1 cells were harvested for protein extraction $48 \mathrm{~h}$ after transfection. Cell protein lysates were used for western blot analysis as previously described (16). The following antibodies were used: anti-BMF (ab9655), antiBBC3 (ab9643), anti-ANGPTL2 (ab35574,) (all from Abcam), anti- $\beta$-actin (sc-47778), goat anti-mouse IgG-HRP (sc-2005) and goat anti-rabbit IgG-HRP (sc-2004) (all from Santa Cruz Biotechnology, Inc., Santa Cruz, CA, USA).

Construction of miR-221 silencing lentivirus. In order to elucidate the role of miR-221 in vivo, we constructed a recombinant lentivirus termed miR-221(D)-LV to generate stable loss-offunction of miR-221 in hepatoma cells. We first prepared a recombinant lentivirus vector named as pmiR-221(D)-LV as previously described (34). Briefly, two oligonucleotides (forward sequence, 5'-CCGGCGAAACCCAGCAGACAAT GTAGCTTTTTTTTGGAAG-3' and reverse sequence, 5'-AATTCTTCCAAAAAAAAGCTACATTGTCTGCTGGG TTTC-3') were chemically synthesized and annealed to form a double-stranded nucleotide with the overhangs (Italic letters in the sequences) of AgeI and EcoRI in 5' and 3' flanking ends, respectively. The bold letters in the forward sequence refer to the complementary sequence to miR-221. This double-stranded nucleotide was directionally cloned into the AgeI/EcoRI- digested lentivirus vector pGCSIL-GFP (GeneChem Co., Ltd., Shanghai, China) to form the recombinant lentivirus vector pmiR-221(D)-LV. pGCSIL-GFP has a polymerase III promoter U6 which can promote the transcription of small non-coding RNAs. The underlined polyT in the 3 ' flanking region of forward sequence is the transcription termination signal for promoter U6. A control recombinant lentivirus vector, pNCGFP-LV, containing a scrambling sequence was also constructed and used as an internal control. All recombinant vectors were verified by sequencing. To obtain high titer recombinant lentivirus, a lentivirus package system (GeneChem Co., Ltd.) was applied, containing three vectors: i) our recombinant lentivirus vector [pmiR-221(D)-LV or pNC-GFPLV], ii) pHelper1.0, and iii) pHelper2.0. pHelper1.0 contains gag gene and pol gene of human immunodeficiency virus (HIV), coding the major structural protein Gag and the virusspecific enzyme Pol of HIV, respectively. pHelper 2.0 contains VSV-G gene of herpes simplex virus (HSV), coding envelope protein needed by virus package. These three vectors were cotransfected into the packaging cell line $293 \mathrm{~T}$ to produce VSV.G-pseudo-typed lentiviral particles. At 8-h post-transfection, the culture media was replaced by complete media. The supernatant of transfected $293 \mathrm{~T}$ was collected at $48 \mathrm{~h}$ posttransfection, and was further concentrated into high titer lentivirus as previously described (35). The titer of lentivirus was evaluated by limiting dilution assay (36).

Animal experiments. BALB/c athymic nude mice (male, 4-6 weeks old, 16-20 g) were purchased from Hubei Research Center of Laboratory Animal (Wuhan, China) and bred in pathogen-free conditions in the Animal Centre of Tongji Medical College. All animals received humane care and all animal experiments were carried out in accordance with the Guide for the Care and Use of Laboratory Animals of Tongji Medical College (Permit no. 130321u). SK-HEP-1 cells were incubated with lentivirus stocks diluted at an MOI of 50 in 


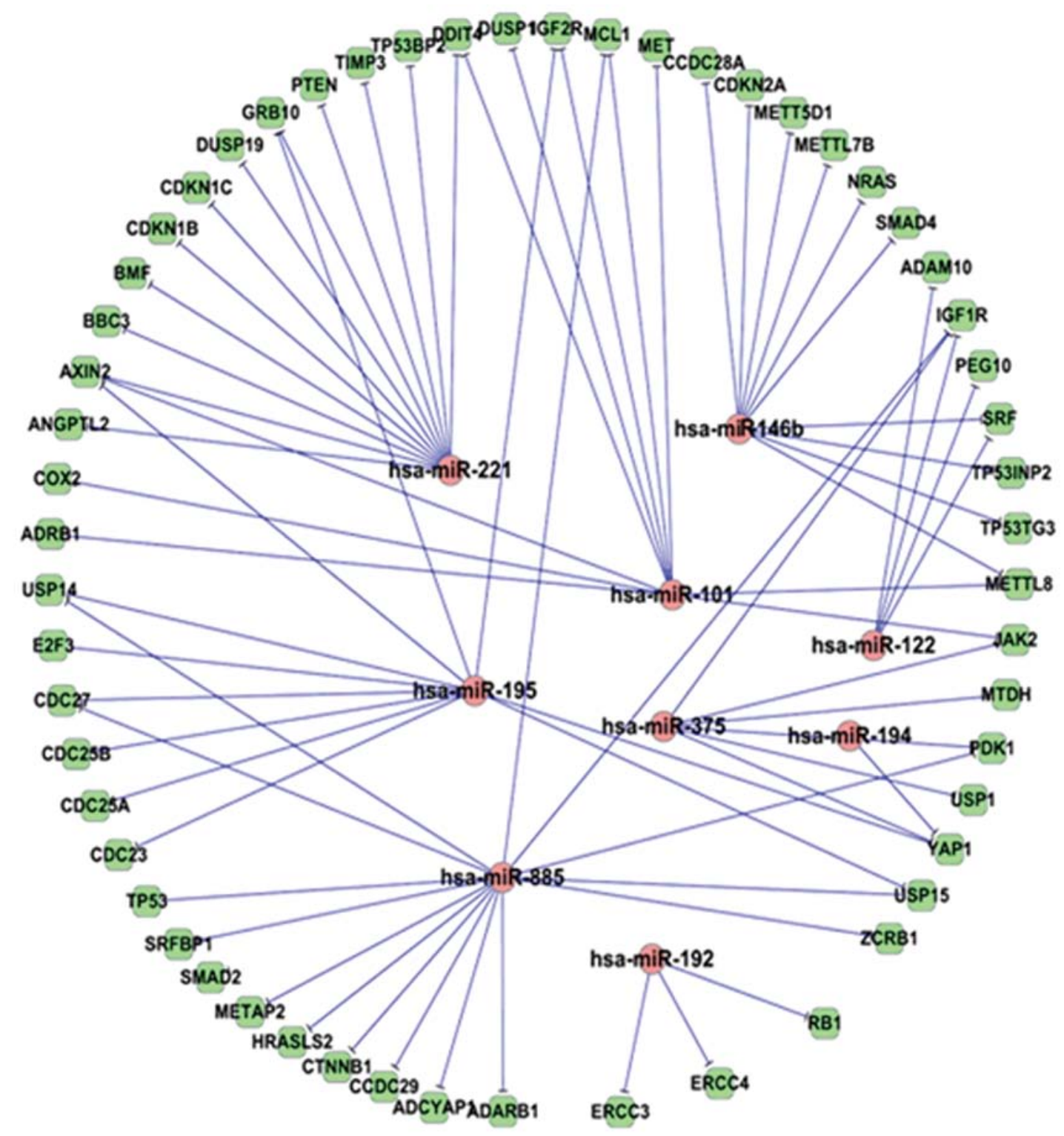

Figure 1. Differentially expressed miRNAs and their targets with HCC genes. The top 9 differentially expressed miRNAs in our miRNA microarray assay were used to predict their targets in published HCC-related genes. Red circle indicates miRNAs, round green rectangle represents HCC genes.

Opti-MEM supplemented with $5 \mu \mathrm{g} / \mathrm{ml}$ polybrene for $10 \mathrm{~h}$. Transduction efficiency was examined by fluorescence microscopy $72 \mathrm{~h}$ later. SK-HEP-1 cells $\left(2 \times 10^{6}\right)$ infected with miR-221(D)-LV or NC-GFP-LV were suspended in $100 \mu 1$ DMEM and injected subcutaneously into the back of mice. Tumor growth was examined every 4 days for $>1$ month. To establish hepatoma xenograft model, $2 \times 10^{6}$ SK-HEP-1 cells were inoculated subcutaneously on the right flank of nude mice. After 8 days, the transplanted nude mice were randomly divided into 2 groups ( $\mathrm{n}=6$ each). TU $\left(2 \times 10^{8}\right)$ miR-221(D)-LV or NC-GFP-LV/animal was administered a single intratumor injection. Tumor volume $(V)$ was monitored by measuring the length $(L)$ and width $(W)$ with vernier caliper and calculated with the formula $V=\left(L \times W^{2}\right) \times 0.5$.

Immunohistochemistry. Resected tumor tissues were fixed in $4 \%$ paraformaldehyde, embedded in paraffin, cut into $4 \mu \mathrm{m}$ pieces and mounted on polylysine-coated slides. Haematoxylin and eosin (H\&E) and Ki67 immunohistochemistry assay were detected as previously described (16).

Statistical analysis. All data are presented as means \pm standard error from 3 separate experiments performed in triplicate except otherwise noted. The differences between groups were analyzed by Student's t-test and $\mathrm{P}<0.05$ was considered to indicate a statistically significant result.

\section{Results}

miR-221 plays a central role in HCC regulatory network. To reveal the regulation of dysregulated miRNAs involved in $\mathrm{HCC}$, bioinformatics analysis was used to predict regulatory targets and TFs-related to hepatocarcinogenesis and construct regulatory networks for dysregulated miRNAs. As shown in Fig. 1, the 9 mostly differentially expressed miRNAs in our miRNA microarray assay (16) were predicted to target 56 


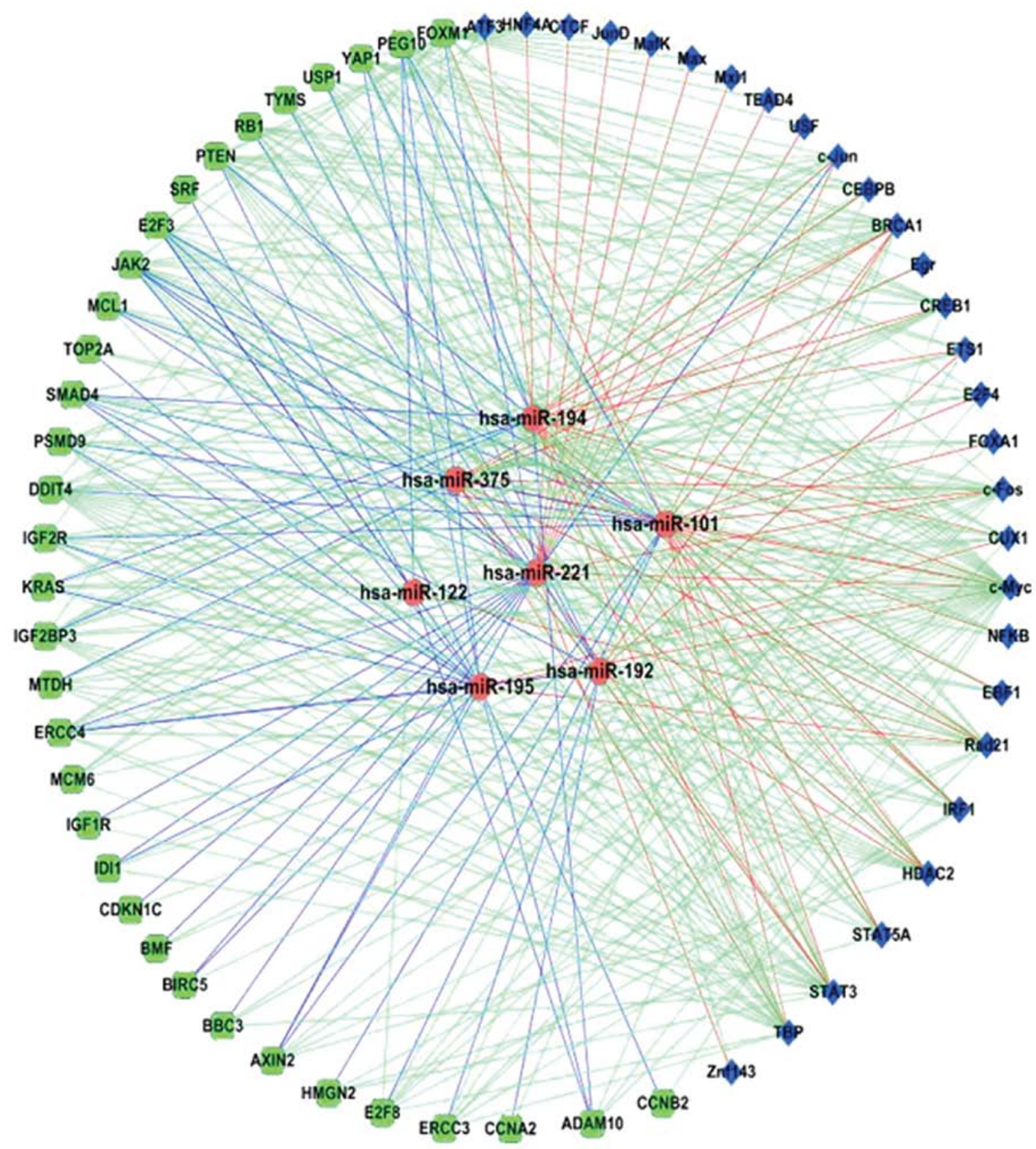

Figure 2. TF and miRNA co-regulatory network in HCC. TF regulation using the encode ChIP-Seq data compiled at UCSC genome browser. Red circle represents miRNAs, green round rectangle is HCC-related genes and blue diamond indicates TF. Blue line indicates the miRNA regulating the HCC-related genes, green line indicates TF regulating HCC-related genes and red line shows the TF regulating miRNA.

HCC-related genes with 73 edges in the HCC miRNA target network. In this network, miR-221 is one of the miRNAs having the most targets of HCC-related genes. Furthermore, since TF is another type of key regulator of gene expression, we also investigated the TFs involved in the HCC regulatory network. Using the ENCODE ChIP-Seq data in UCSC genome browser, we obtained the TF targets in the HCC genes and miRNAs. Choosing those TFs involved in HCC and using the same strategy as described in our previous study (13), we constructed the co-regulatory network among the HCC-related TFs, genes and miRNAs (Fig. 2). Fig. 2 shows that there are many FFLs among TFs, miRNAs and HCC genes. In an FFL, the TF regulates miRNA and HCC gene, while the miRNA also regulates the same HCC gene. This network contains
332 regulatory relations (edges) among 70 molecules (nodes), including 7 miRNAs, 29 TFs and 34 HCC-related genes. As shown in this network, miR-221 is a core miRNA regulating many HCC genes and being regulated by many TFs, while c-Myc, c-Jun and STAT are core TFs. Notably, FOXM1 is an HCC-related gene and also a TF. Thus, in the network, it regulates other HCC-related genes or miRNAs and also as a target of other TFs or miRNAs. Since miR-221 is the most highly expressed miRNA in our microarray results and it is also a core miRNA in our regulatory networks, we then focused on miR-221. Extracting miR-221-related targets and TFs in Fig. 2 co-regulatory network and adding some other known miR-221 targets, we obtained the miR-221 regulatory network (Fig. 3). As shown in Fig. 3, there are many FFL regulations particu- 


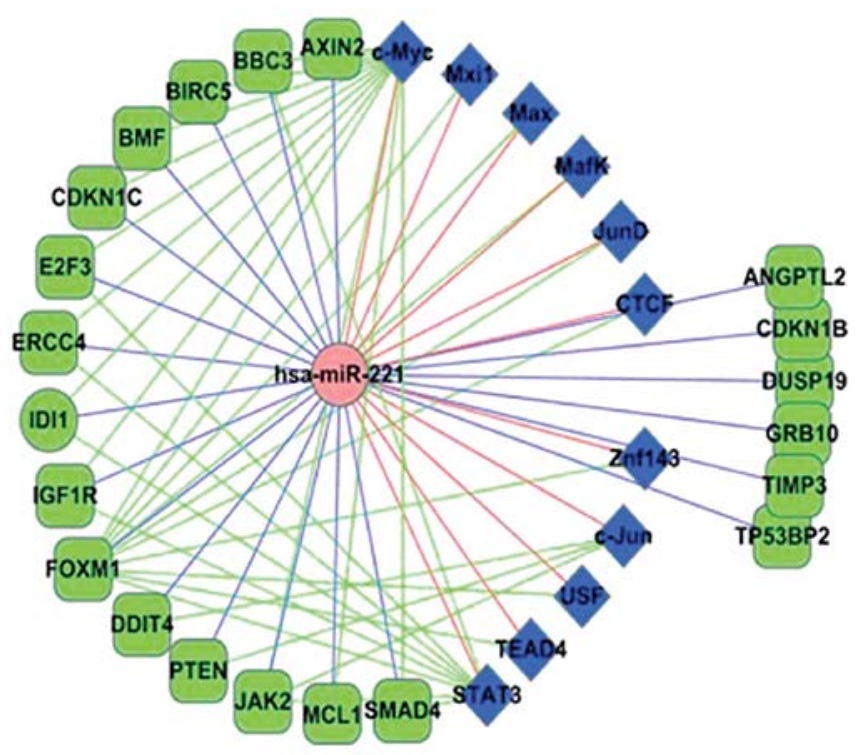

Figure 3. The regulatory network of miR-221 and its related TFs in HCC genes. A subset data specific for miR-221 extracted from the Figs. 1 and 2. Red circle represents miRNA, green round rectangle is HCC-related genes and blue diamond indicates TF. Blue line indicates the miRNA regulating the HCC-related genes, green line indicates TF regulating HCC-related genes and red line shows the TF regulating miRNA. c-Myc and STAT3 are the top two transcription factors targeting to most HCC genes and forming feedforward loops with miR-221.

larly among the c-Myc, miR-221 and HCC-related genes. These may indicate that c-Myc plays an important role in the miR-221 functional pathway in HCC development. These analyses indicate that miR-221 is a critical modulator of HCC and could be considered as a potential therapeutic target.

miR-221 silencing inhibits tumorigenic properties of liver cancer cells in vitro. To assess the role of miR-221 in hepatocarcinogenesis, we used gain- and loss-of-function methods. As shown in Fig. 4A, the increase of miR-221 or inhibition of miR-221 expression were verified by TaqMan qRT-PCR in SK-HEP-1, HepG2, SMMC-7721 or HeLa cells transfected with pre-miR-221 or anti-miR-221.

Sustained cell proliferation is the most distinctive property of cancer. Inhibiting miR-221 markedly suppressed the proliferation of liver cancer cells (Fig. 4B). To examine whether compromised cell proliferation could be attributed to the cell cycle arrest, we further analyzed the status of cell cycle. As shown in Fig. 4C, miR-221 silencing significantly increased the percentages of cells in G1 phase, which indicated the occurrence of G1 arrests in treated SK-HEP-1 cells, while the cell percentages in $\mathrm{S}$ phase were obviously shrunk by nearly $10 \%$. Escaping from cell apoptosis is another advantage of tumor cells for overwhelming growth. The result from Annexin V/PI combined labeling flow cytometry analysis showed that the numbers of apoptotic cells were clearly increased in SK-HEP-1 cells treated with anti-miR-221 (Fig. 4D). To assess the functional role of miR-221 in tumor formation, the capacity of colony formation and anchorageindependent growth were measured in SK-HEP-1 transfected with anti-miR-221. Notably, anti-miR-221-transfected cells displayed much fewer and smaller colonies than control trans- fectants (Fig. 4E). Metastasis is another hallmark of cancer. As shown in Fig. 4F, miR-221 inhibition effectively suppressed the ability of SK-HEP-1 cell migration and invasion.

These data indicate that miR-221 functions as an oncogene and its silencing inhibits tumorigenic properties of liver cancer cells in vitro.

miR-221 targets BMF, BBC3 and ANGPTL2. To elucidate the tumorigenic role of miR-221 in $\mathrm{HCC}$, we further verified several putative targets of miR-221 from Fig. 3 (Fig. 5A). As shown in Fig. 5B, luciferase assay indicated that miR-221 could directly aim at its predicted binding sites of BMF, BBC3 and ANGPTL2, leading to the suppression of luciferase expression, whereas when the binding site was mutated by site-specific mutagenesis, the luciferase activity was reserved comparable with the matched negative control group. At the mRNA level, miR-221 inhibition elicited an obvious upregulation of ANGPTL2 but not of BMF and BBC3 (Fig. 5C). While at the protein level, BMF, BBC3 and ANGPTL2 protein were apparently increased in anti-miR-221-treated cells (Fig. 5D). These data suggest that miR-221 may suppress its targets BMF, BBC3 and ANGPTL2 mainly through a translational inhibition at the protein level with or without mRNA degradation.

Lentivirus-mediated-anti-miR-221 inhibits tumor formation and growth of hepatoma xenografts in vivo. Given the importance of miR-221 in HCC carcinogenesis, it is not surprising that miR-221 is an attractive target for developing novel therapies. Lentiviral vectors are the most recently developed virus-derived vectors for gene therapy applications, and have demonstrated the ability to transduce dividing and non-dividing cells, and sustain long-term transgene expression, which makes them uniquely attractive as gene therapy vectors (37). Thus, we successfully constructed a recombinant lentiviral vector termed miR-221(D)-LV (and NC-GFP-LV as control) to transduce SK-HEP-1 cells (Fig. 6A) and inhibit the expression of miR-221 (Fig. 6B-a). First, we employed SK-HEP-1 cells pre-infected with miR-221(D)-LV to establish subcutaneous tumors in nude mice, and measured the tumor growth and significant features. As depicted in Fig. 6C, depletion of miR-221 in vitro by miR-221(D)-LV renders SK-HEP-1 cells less efficient in establishing tumors in vivo. The average fold increase of tumor volumes at the sacrifice with respect to the first measurements was much smaller in miR-221(D)-LV pre-infected tumors vs. control tumors $(9.05 \pm 1.62$ vs. $16.23 \pm 2.89, \mathrm{P}=0.034)$. Next, we further assessed the effects of miR-221 inhibition on pre-established SK-HEP-1 xenografts. Consistent with our expectation, a single intratumor injection with $2 \times 10^{8}$ TU miR-221(D)-LV on day 8 (when the tumor volume reached $\sim 80 \mathrm{~mm}^{3}$ ), reduced the growth of tumors. As shown in Fig. 6D, the growth curves of miR-221(D)-LV and NC-GFP-LV-treated tumors became divergent on day 16 after the inoculation of SK-HEP-1 in mice, and this trend became more obvious and continued to the end of the experiment. Relative to the first measurements on day 8 , the average fold increase of tumor volumes at the sacrifice of miR-221(D)LV-treated groups was significantly smaller than that of the negative control $(7.81 \pm 1.02$ vs. $12.61 \pm 1.37, \mathrm{P}=0.041)$.

To clarify the cellular mechanisms underlying miR-221(D)LV mediated tumor suppression, resected tissues from those 

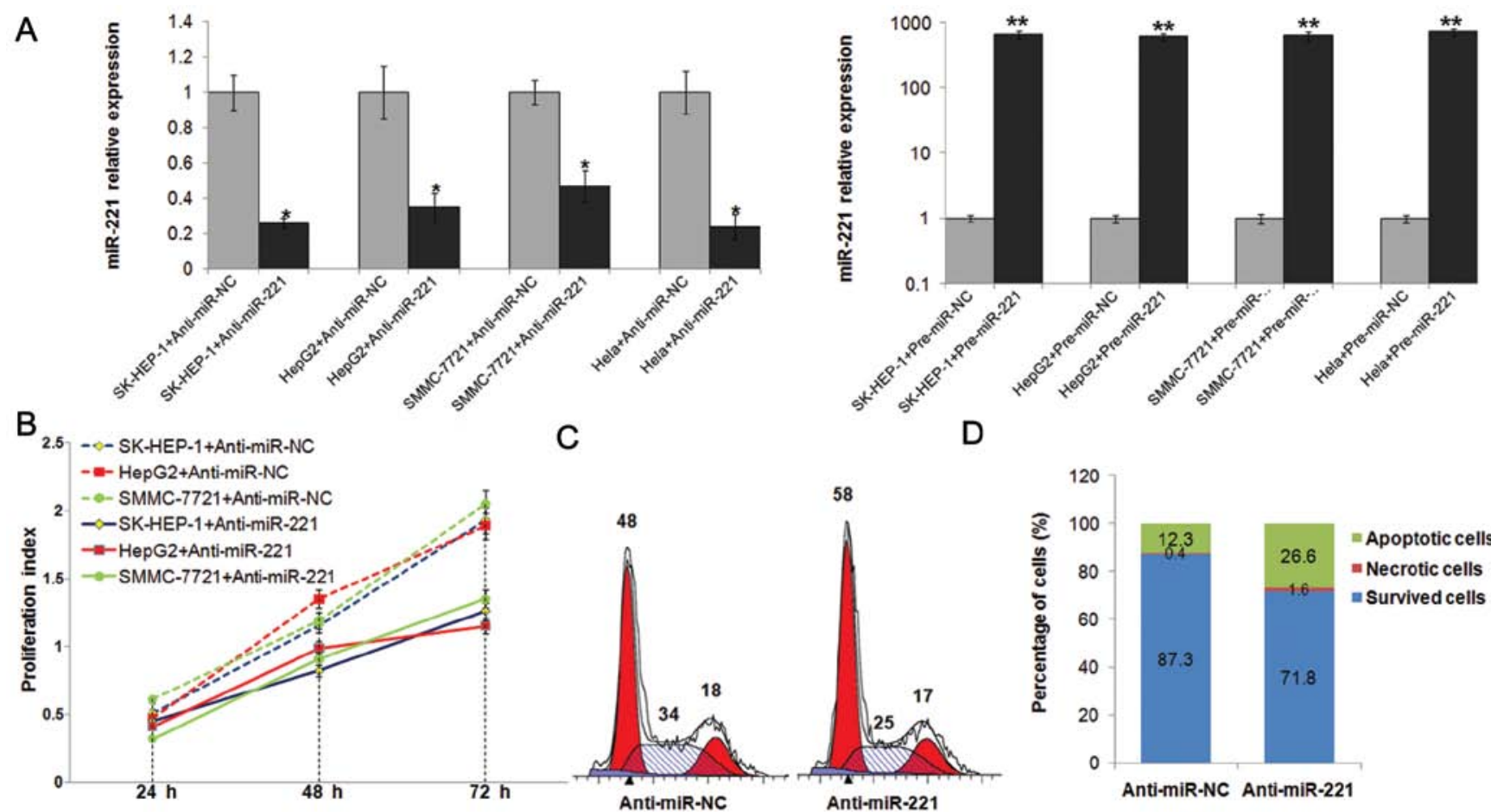

C

D

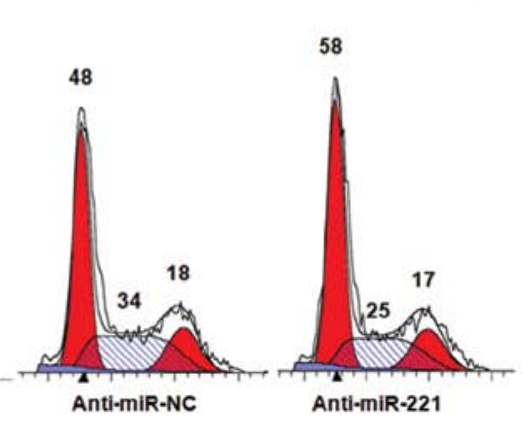

$\mathrm{F} \mathrm{a}$

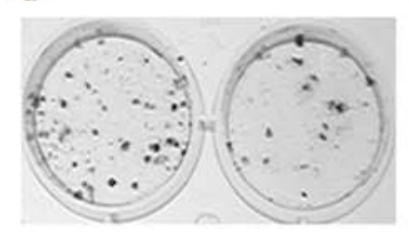

Anti-miR-NC

c

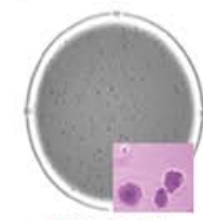

Anti-miR-NC

Anti-miR-221

b

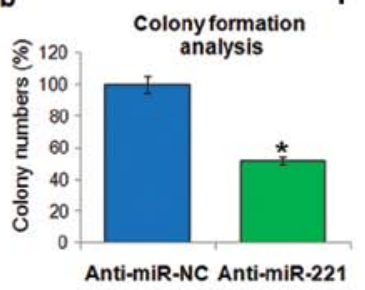

d

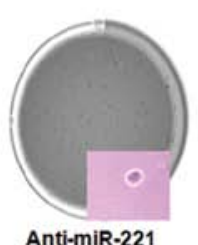

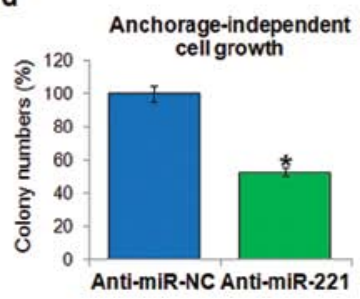
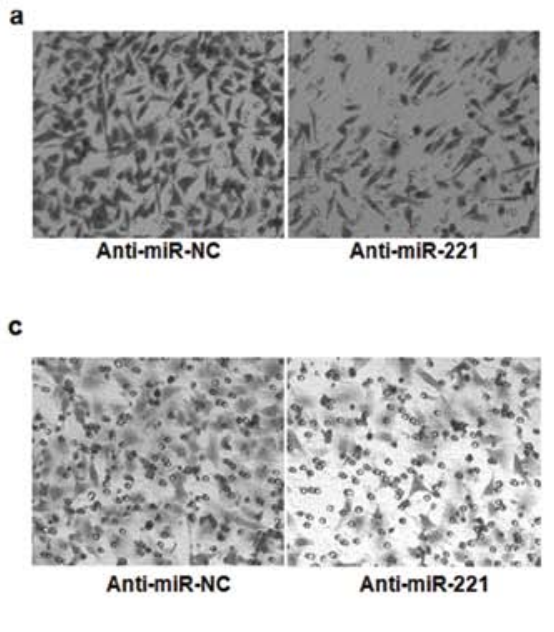
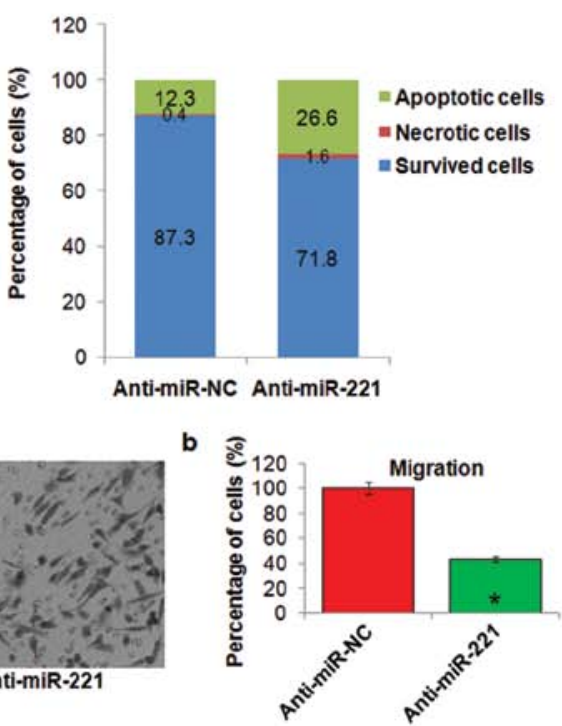

d

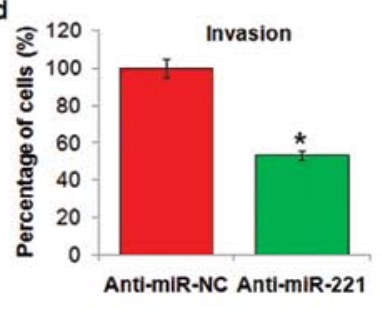

Figure 4. miR-221 silencing inhibits tumorigenic properties of hepatocarcinoma. (A) Relative expression of miR-221 was detected by TaqMan qRT-PCR in various hepatoma cell lines or HeLa cell line transfected with anti-miR-221, pre-miR-221 or their matched negative control. The relative miRNA expression from the negative control group was designated as 1. (B) miR-221 inhibition suppresses cell proliferation in SK-HEP-1, HepG2 and SMMC-7721 cells. (C) Cell cycle determined by propidium iodide (PI) staining flow cytometry. Numbers over each histogram indicate the percentage of cells in G0-G1, S and G2-M cell-cycle phases. (D) Cell apoptosis detected by Annexin V/PI combined labeling flow cytometry. Apoptotic evaluation was determined by the percentage of apoptotic cell number in total cell number. (E-a and -c) Representative results of colony formation and anchorage-independent growth in soft agar using the indicated clones. (E-b and -d) Histogram indicated that anti-miR-221 markedly inhibited the colony formation and anchorage-independent cell growth. The percentage of colony numbers from the negative control group was designated as $100 \%$. (F) Anti-miR-221 reduced the cell migration and invasion ability. (a and c) Results of cell invasion and migration across an $8-\mu \mathrm{m}$ pore size membrane with or without Matrigel. (b and d) Histogram reported the percentage of cells across a membrane with $8-\mu \mathrm{m}$ pores with or without Matrigel after transfection with anti-miR-221 or its matched control. The percentage of cells for migration or invasion of the negative control group was designated as $100 \%$. ${ }^{*} \mathrm{P}<0.05$ and ${ }^{* *} \mathrm{P}<0.01$.

treated xenograft tumors were analyzed to verify miR-221 expression, and measured for mitotic index and expression of Ki67 as markers of proliferation. At the end of the experiment, miR-221(D)-LV effectively reduced the expression of miR-221 (Fig. 6B-b). Consistent with the above results, HE and Ki67 staining revealed markedly reduced proliferation index in miR-221(D)-LV-treated tumors (Fig. 6E and F). These results indicate that miR-221 silencing inhibits liver cancer cell growth in vivo.

\section{Discussion}

The rationale for using miRNAs as potential therapeutic targets for cancer is based on many facts: i) miRNAs are natural antisense interactors; ii) miRNA expression profiles have been shown to be related to disease state and treatment response and the dysregulated miRNAs contribute to the initiation and development of cancer; iii) the small size (22-24 nucleotides in length) makes them very attractive for drug development (38). 
A

\begin{tabular}{|c|c|}
\hline & $\begin{array}{l}\text { predicted consequential pairing of target } \\
\text { region (top) and miRNA (bottom) }\end{array}$ \\
\hline $\begin{array}{l}\text { Position 3683-3689 of BMF 3' UTR } \\
\text { hsa-miR-221 }\end{array}$ & 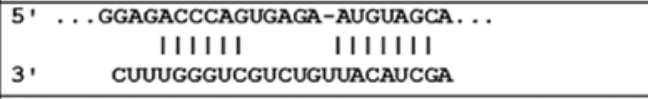 \\
\hline $\begin{array}{l}\text { Position 95-101 of BBC3 3' UTR } \\
\text { hsa-miR-221 }\end{array}$ & $\begin{array}{c}5, \ldots \text { GACUUUCUCUGCACC--AUGUAGCA } \ldots \\
\text { I I I I I I I I I I } \\
3, \quad \text { CUUUGGGUCGUCUGUUACAUCGA }\end{array}$ \\
\hline $\begin{array}{l}\text { Position 1488-1494 of ANGPTL2 3' UTR } \\
\text { hsa-miR-221 }\end{array}$ & $\begin{array}{c}5, \ldots \text { CUCACAAAGUGUACCAUGUAGCA. } . . \\
\text { IIIIIIII } \\
3, \quad \text { CUVUGGGUCGUCUGUUACAUCGA }\end{array}$ \\
\hline
\end{tabular}

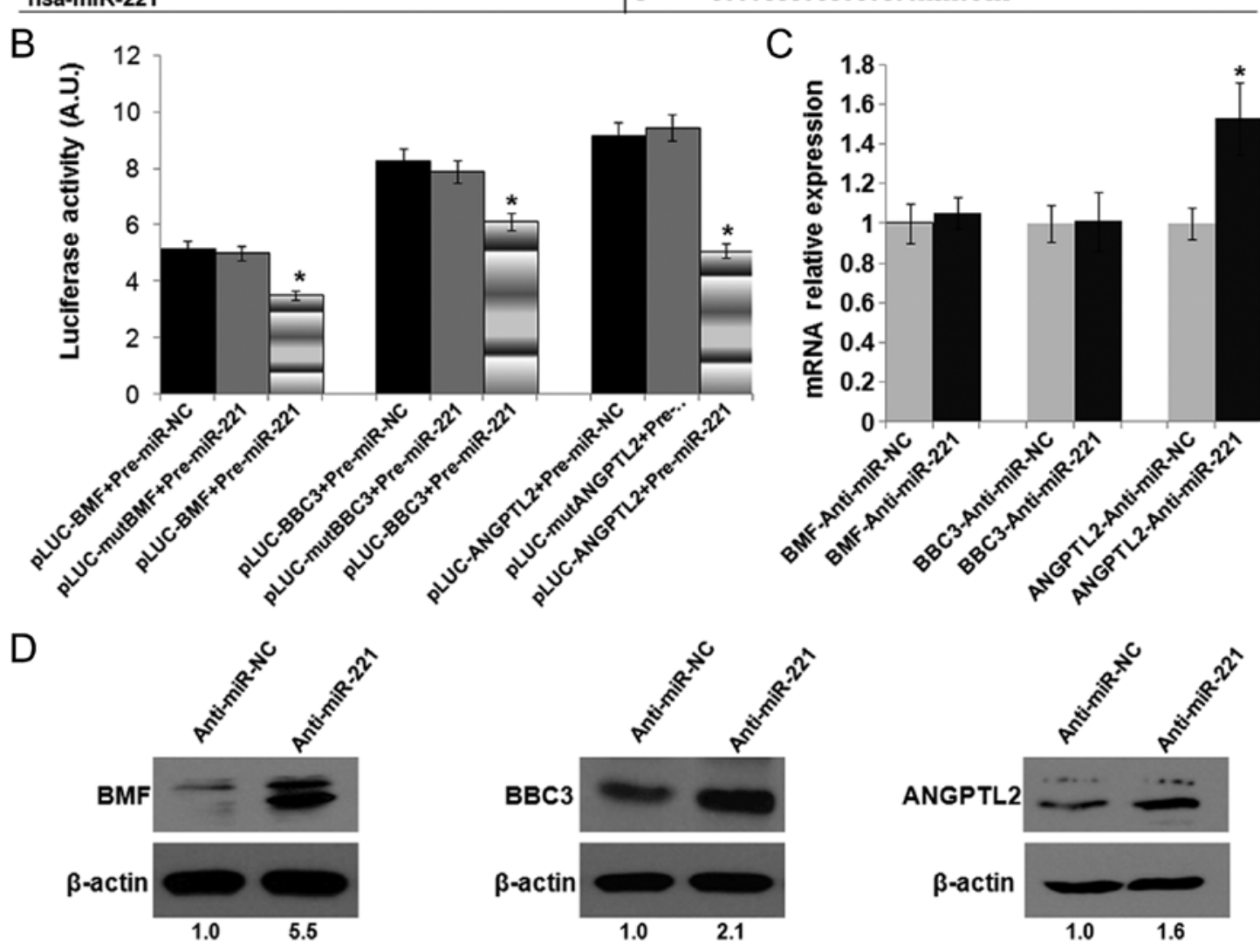

Figure 5. Target validation of miR-221 in hepatocellular carcinoma. (A) Predicted consequential pairing of target region and miR-221 by TargetScan. (B) Luciferase assay in SK-HEP-1 cells. pLUC-target vector was cotransfected with pre-miR-221 or pre-miR-NC. Relative repression of luciferase expression was standardized to $\beta$-gal signal. Luciferase activity displayed a significant decrease following miR-221 enforced expression compared with control groups. (C) The target expressions at mRNA level were measured by SYBR-Green qRT-PCR after inhibition of miR-221 in SK-HEP-1. ANGPTL-2 but not BMF and BBC3 mRNA was upregulated by anti-miR-221. The relative mRNA expression from negative control group was designated as 1 . (D) Western blot analyses showed increased BMF, BBC3, ANGPTL-2 protein expression after inhibition of miR-221 expression. " $\mathrm{P}<0.05$.

In the present study, we found that miR-221 plays a central role in HCC signaling pathway by bioinformatics analysis. It is common in biology for gene expression and important processes to have multiple layers of regulation and control. TFs and miRNAs are key regulators of gene expression at the transcription and post-transcription level. They may regulate the same target gene and regulate mutually to form complex regulatory module and network including FFLs, thus carry out the subtle regulation of the target gene expression (39). It has been predicted that there are hundreds of FFLs in human genome (40) and some of them have been experimentally verified such as TP53/miR-106b/E2F (15) and NF- $\mathrm{kB} / \mathrm{miR}-19 / \mathrm{CYLD}$ (13) in cancer cell proliferation. Many TFs and miRNAs have been reported to be involved in the progress of $\mathrm{HCC}$, which is a complex process (41). Therefore, the TF and miRNA regulations will be the key regulation of HCC progress. In this study, we started from the top differentially expressed miRNAs and constructed the TF-miRNA co-regulatory FFLs in HCC using the same strategy we used for schizophrenia and T-cell acute lymphoblastic leukemia $(13,42)$. Figs. 1-3 show that miR-221 was a core miRNA with the most targets of HCC-related genes and formed many FFL regulations with some key TFs. We also showed that miR-221 may target CDKN1B, CDKN1C, BMF, DDIT4, PTEN, TIMP3, E2F3, TP53BP2, BBC3, ANGPTL2 and many other genes related to HCC (Fig. 3). Some of these targets have been experimentally confirmed. Furthermore, Fig. 3 also provides insight into FFL regulation of miR-221 involved in HCC. TFs c-Myc, c-Jun and STAT3 were predicted to target many important HCC genes and formed FFLs with miR-221. Particularly, c-Myc and miR-221 formed 13 FFLs with HCC genes, including FFLs c-Myc/miR-221/E2F3 and c-Myc/miR-221/CDKN1C. c-Myc is a widely studied 


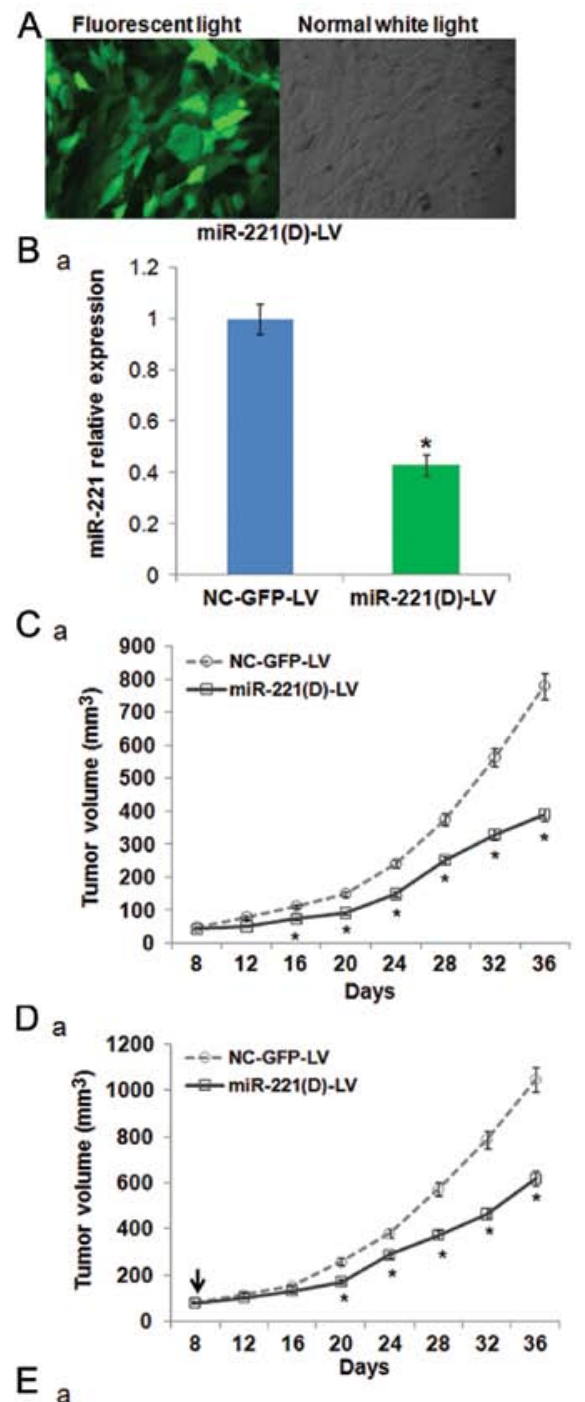

E a

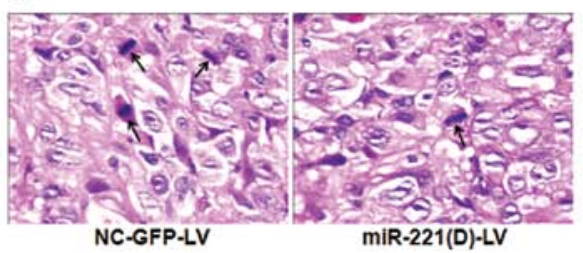

$\mathrm{F}$ a

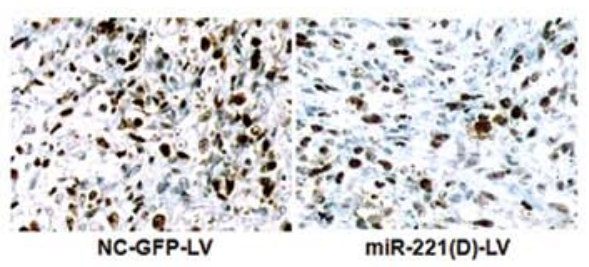

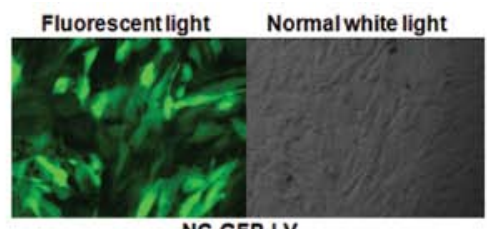

NC-GFP-LV

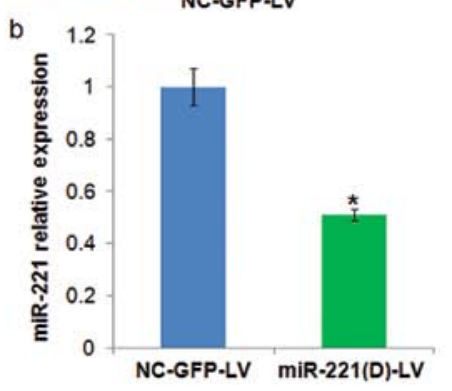

b 25

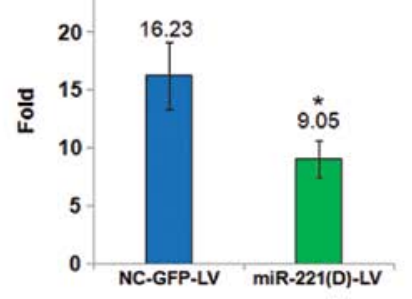

b
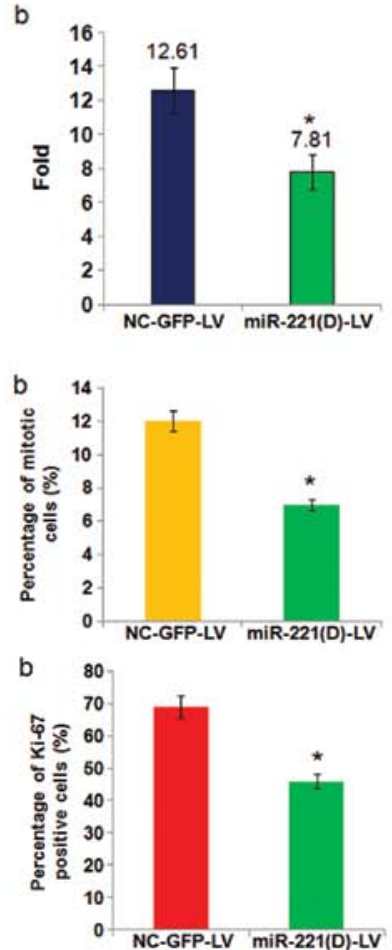

Figure 6. miR-221 silencing slows down the tumor formation and growth of hepatoma xenografts. (A) Fluorescent microscopy showed efficient transduction of SK-HEP-1 by lentivirus, as indicated by eGFP expression, 15 days following lentiviral vector administration. (B) Relative expression of miR-221 was detected by TaqMan qRT-PCR. (B-a) SK-HEP-1 cells pre-infected with recombinant lentiviral vector miR-221(D)-lv (96 h after transduction) significantly inhibited the expression of miR-221 in vitro; (B-b) verified inhibition of miR-221 expression by intratumor injection one time with miR-221(D)-lv on pre-established SK-HEP-1 xenografts in vivo. The relative miRNA expression from the negative control group was designated as 1 . " $\mathrm{P}<0.05$. (C) In vivo tumor growth of recombinant lentivirus pre-infected SK-HEP-1 xenograft in nude mice. (C-a) Average tumor volumes were represented ( $\mathrm{n}=6$ for both experimental groups) starting from day 8 when tumor volumes were $\sim 40 \mathrm{~mm}^{3}$ until the last measurement before being sacrificed on day 36; (C-b) histogram indicated the average fold increase of tumor volumes at the sacrifice with respect to the first measurements and miR-221(D)-LV-treated groups was significantly smaller than negative control. (D) Inhibition of miR-221 expression on pre-established SK-HEP-1 xenograft growth by intratumor injection one time with miR-221(D)-LV. (D-a and -b) Tumor growth curves and average volume fold increase of tumors at the sacrifice with respect to the first measurements. (E-a) Haematoxylin and eosin stained section (magnification, $\mathrm{x}$ 400). SK-HEP-1 xenografts treated with miR-221(D)-LV had low mitotic index compared with the negative control group; arrows indicate mitotic images. (E-b) Graph of the mitotic index as percent of positive cells (8 fields, 3 sections for each tumor). (F-a) Immunohistochemistry of the proliferation marker Ki-67 in tumor tissue indicated that SK-HEP-1 xenografts treated with miR-221(D)-LV had markedly reduced Ki-67-positive cells; cells with brown nuclear staining were considered to be positive for Ki-67 (magnification, x200). (F-b) Graph of Ki-67 expression as percent of positive cells ( 8 fields, 3 sections for each tumor). 
oncogene and it plays a critical role in human pathogenesis (43). Thus, the feed-forward loop regulatory modules among c-Myc, miR-221 and HCC genes warrant further exploration.

Previous studies have demonstrated that overexpression of miR-221 augments cell proliferation, colony formation, invasion and increase the number of cells in $S$ phase, while inhibiting cell apoptosis $(8,17,18,26,27)$. Here, we re-confirmed the role of miR-221 as an oncogene through miR-221 silencing functional analysis including cell proliferation, cell cycle, apoptosis, cell migration, invasion and clone formation and found that inhibition of miR-221 in liver cancer cells decreased cell proliferation, clonogenicity, migration/invasion and also induced G1 arrest and apoptosis (Fig. 4). To investigate the molecular mechanism of miR-221-mediated phenotype in hepatoma cells described above, we further validated that BMF, BBC3 and ANGPTL2 are direct targets of miR-221 and showed that miR-221 suppressed the expression of BMF, BBC3 and ANGPTL2 by binding directly to the 3'-UTR of these genes (Fig. 5), which support the findings of our bioinformatics analysis. Among them, BMF is a member of the Bcl-2 family belonging to pro-apoptotic $\mathrm{BH} 3$-only proteins. Our result that BMF is a target of miR-221 is in accordance with the result published by Gramantieri et al (27). BBC3, also known as PUMA, is an essential mediator of cell death in response to various apoptotic signals, notable by its pivotal role in p53-induced apoptotic pathway (44). Markedly, Pineau et al found that BBC3 is a putative target of miR-221 and that there is an inverse correlation between miR-221 and $\mathrm{BBC} 3$ expression in $\mathrm{HCC}$, but failed to validate $\mathrm{BBC} 3$ as a direct target of miR-221 by luciferase assay in the supporting information of their publication (8). However, we have verified that BBC3 is a direct target of miR-221, and this is similar to the results observed in human epithelial cancers and glioblastoma $(45,46)$. ANGPTL2 belongs to the angiopoietin family for its limited sequence homology with angiopoietins (47). It has been reported that ANGPTL2 works as a functional tumor suppressor gene repressing cell growth and impairing cell clone formation in ovarian cancer (48). To the best of our knowledge, that miR-221 directly targets ANGPTL2 has not previously been reported. These results indicate that miR-221 silencing could inhibit liver cancer malignant properties in vitro through regulating many HCC-related genes including BMF, BBC3 and ANGPTL2.

The above findings strongly underscore the possibility of miR-221 as an ideal target for therapeutic intervention. In Fig. 6, we showed that in vitro depletion of miR-221 by lentivirusmediated-anti-miR-221 renders liver cancer cells less efficient in the establishment of in vivo xenografts, while in vivo intratumoral knockdown of miR-221 reduces tumor growth of liver cancer cell xenografts. In the present study, lentiviral vectors provided an efficient gene delivery and gave us a stable loss of function of miR-221 for study in vitro and in vivo. Hence, lentivirus-mediated antagomir expression for stable loss of function of a specific miRNA may be a useful laboratory tool to study miRNA functions and may be considered for clinical gene therapy applications $(34,49)$. Our animal studies indicated that lentivirus-mediated-anti-miR-221 treatment could suppress the growth of hepatoma xenografts in vivo. Similarly, Park et al showed miR-221 silencing by chol-anti-miR-221 blocks HCC and promotes survival in a valid orthotopic mouse model of HCC (50). These results support that miR-221 may be an ideal target for HCC therapy and future studies to determine the relative efficacy of targeting miR-221 compared with other miRNA delivery methods, such as nanoparticles, may be required to identify the optimal miRNA delivery methods for further clinical translation.

In conclusion, we demonstrated that miR-221 is a critical modulator in HCC and miR-221 silencing could inhibit liver cancer malignant properties in vitro and in vivo through regulation of its targets including BMF, BBC3, ANGPTL2, emphasizing the promising potential of miR-221 inhibition for HCC therapy.

\section{Acknowledgements}

This study was supported by grants from the National Natural Science Foundation of China (nos. 81101824, 31171271, 81000874 and 81070333), the Outstanding Youth Science Foundation of Tongji Hospital (no. YXQN005), and the Youth Sciences and Technology Chenguang Planning of Wuhan (no. 2014070404010219).

\section{References}

1. Roberts LR: Sorafenib in liver cancer - just the beginning. N Engl J Med 359: 420-422, 2008.

2. Murakami Y, Yasuda T, Saigo K, et al: Comprehensive analysis of microRNA expression patterns in hepatocellular carcinoma and non-tumorous tissues. Oncogene 25: 2537-2545, 2006.

3. Varnholt H, Drebber U, Schulze F, et al: MicroRNA gene expression profile of hepatitis $\mathrm{C}$ virus-associated hepatocellular carcinoma. Hepatology 47: 1223-1232, 2008.

4. Ji J, Shi J, Budhu A, et al: MicroRNA expression, survival, and response to interferon in liver cancer. N Engl J Med 361: 1437-1447, 2009.

5. Ladeiro Y, Couchy G, Balabaud C, et al: MicroRNA profiling in hepatocellular tumors is associated with clinical features and oncogene/tumor suppressor gene mutations. Hepatology 47: 1955-1963, 2008

6. Gramantieri L, Ferracin M, Fornari F, et al: Cyclin G1 is a target of miR-122a, a microRNA frequently down-regulated in human hepatocellular carcinoma. Cancer Res 67: 6092-6099, 2007.

7. Hou J, Lin L, Zhou W, et al: Identification of miRNomes in human liver and hepatocellular carcinoma reveals miR-199a/b$3 p$ as therapeutic target for hepatocellular carcinoma. Cancer Cell 19: 232-243, 2011.

8. Pineau P, Volinia S, McJunkin K, et al: miR-221 overexpression contributes to liver tumorigenesis. Proc Natl Acad Sci USA 107: 264-269, 2010.

9. Yuen MF, Wu PC, Lai VC, Lau JY and Lai CL: Expression of c-Myc, c-Fos, and c-jun in hepatocellular carcinoma. Cancer 91: 106-112, 2001.

10. Kawate S, Fukusato T, Ohwada S, Watanuki A and Morishita Y: Amplification of $c$-myc in hepatocellular carcinoma: correlation with clinicopathologic features, proliferative activity and p53 overexpression. Oncology 57: 157-163, 1999.

11. Lin CP, Liu CR, Lee CN, Chan TS and Liu HE: Targeting c-Myc as a novel approach for hepatocellular carcinoma. World J Hepatol 2: 16-20, 2010.

12. Suzuki H, Fujita H, Mullauer L, et al: Increased expression of c-jun gene during spontaneous hepatocarcinogenesis in LEC rats. Cancer Lett 53: 205-212, 1990.

13. Ye H, Liu X, Lv M, et al: MicroRNA and transcription factor co-regulatory network analysis reveals miR-19 inhibits CYLD in T-cell acute lymphoblastic leukemia. Nucleic Acids Res 40: 5201-5214, 2012.

14. Yu Z, Wang C, Wang M, et al: A cyclin D1/microRNA 17/20 regulatory feedback loop in control of breast cancer cell proliferation. J Cell Biol 182: 509-517, 2008.

15. Brosh R, Shalgi R, Liran A, et al: p53-Repressed miRNAs are involved with $\mathrm{E} 2 \mathrm{~F}$ in a feed-forward loop promoting proliferation. Mol Syst Biol 4: 229, 2008. 
16. He XX, Chang Y, Meng FY, et al: MicroRNA-375 targets AEG-1 in hepatocellular carcinoma and suppresses liver cancer cell growth in vitro and in vivo. Oncogene 31: 3357-3369, 2012.

17. Garofalo M, Di Leva G, Romano G, et al: $m i R-221 \& 222$ regulate TRAIL resistance and enhance tumorigenicity through PTEN and TIMP3 downregulation. Cancer Cell 16: 498-509, 2009.

18. le Sage C, Nagel R, Egan DA, et al: Regulation of the $\mathrm{p} 27^{\mathrm{Kip} 1}$ tumor suppressor by miR-221 and miR-222 promotes cancer cell proliferation. EMBO J 26: 3699-3708, 2007.

19. Ciafre SA, Galardi S, Mangiola A, et al: Extensive modulation of a set of microRNAs in primary glioblastoma. Biochem Biophys Res Commun 334: 1351-1358, 2005.

20. Lee EJ, Gusev Y, Jiang J, et al: Expression profiling identifies microRNA signature in pancreatic cancer. Int J Cancer 120 1046-1054, 2007.

21. Gottardo F, Liu CG, Ferracin M, et al: Micro-RNA profiling in kidney and bladder cancers. Urol Oncol 25: 387-392, 2007.

22. He H, Jazdzewski K, Li W, et al: The role of microRNA genes in papillary thyroid carcinoma. Proc Natl Acad Sci USA 102: 19075-19080, 2005.

23. Chun-Zhi Z, Lei H, An-Ling Z, et al: MicroRNA-221 and microRNA-222 regulate gastric carcinoma cell proliferation and radioresistance by targeting PTEN. BMC Cancer 10: 367, 2010.

24. Sun K, Wang W, Zeng JJ, Wu CT, Lei ST and Li GX: MicroRNA221 inhibits CDKN1C/p57 expression in human colorectal carcinoma. Acta Pharmacol Sin 32: 375-384, 2011.

25. Callegari E, Elamin BK, Giannone F, et al: Liver tumorigenicity promoted by microRNA-221 in a mouse transgenic model. Hepatology 56: 1025-1033, 2012.

26. Fornari F, Gramantieri L, Ferracin M, et al: MiR-221 controls CDKN1C/p57 and CDKN1B/p27 expression in human hepatocellular carcinoma. Oncogene 27: 5651-5661, 2008.

27. Gramantieri L, Fornari F, Ferracin M, et al: MicroRNA-221 targets $\mathrm{Bmf}$ in hepatocellular carcinoma and correlates with tumor multifocality. Clin Cancer Res 15: 5073-5081, 2009.

28. Lewis BP, Shih IH, Jones-Rhoades MW, Bartel DP and Burge CB Prediction of mammalian microRNA targets. Cell 115: 787-798, 2003.

29. John B, Enright AJ, Aravin A, Tuschl T, Sander C and Marks DS: Human MicroRNA targets. PLoS Biol 2: e363, 2004.

30. Sethupathy P, Corda B and Hatzigeorgiou AG: TarBase: a comprehensive database of experimentally supported animal microRNA targets. RNA 12: 192-197, 2006.

31. Jiang Q, Wang Y, Hao Y, et al: miR2Disease: a manually curated database for microRNA deregulation in human disease. Nucleic Acids Res 37: D98-D104, 2009.

32. Landt SG, Marinov GK, Kundaje A, et al: ChIP-seq guidelines and practices of the ENCODE and modENCODE consortia. Genome Res 22: 1813-1831, 2012.

33. Shannon P, Markiel A, Ozier O, et al: Cytoscape: a software environment for integrated models of biomolecular interaction networks. Genome Res 13: 2498-2504, 2003.

34. Scherr M, Venturini L, Battmer K, et al: Lentivirus-mediated antagomir expression for specific inhibition of miRNA function. Nucleic Acids Res 35: e149, 2007.
35. Scherr M, Battmer K, Ganser A and Eder M: Modulation of gene expression by lentiviral-mediated delivery of small interfering RNA. Cell Cycle 2: 251-257, 2003.

36. Naldini L, Blömer U, Gallay P, et al: In vivo gene delivery and stable transduction of nondividing cells by a lentiviral vector. Science 272: 263-267, 1996.

37. D'Costa J, Mansfield SG and Humeau LM: Lentiviral vectors in clinical trials: current status. Curr Opin Mol Ther 11: 554-564, 2009.

38. Tili E, Michaille JJ, Gandhi V, Plunkett W, Sampath D and Calin GA: miRNAs and their potential for use against cancer and other diseases. Future Oncol 3: 521-537, 2007.

39. Hobert O: Gene regulation by transcription factors and microRNAs. Science 319: 1785-1786, 2008.

40. Re A, Corá D, Taverna D and Caselle M: Genome-wide survey of microRNA-transcription factor feed-forward regulatory circuits in human. Mol Biosyst 5: 854-867, 2009.

41. Zeng L, Yu J, Huang T, et al: Differential combinatorial regulatory network analysis related to venous metastasis of hepatocellular carcinoma. BMC Genomics 13 (Suppl 8): S14, 2012.

42. Guo AY, Sun J, Jia P and Zhao Z: A novel microRNA and transcription factor mediated regulatory network in schizophrenia. BMC Syst Biol 4: 10, 2010.

43. Zimonjic DB and Popescu NC: Role of DLC1 tumor suppressor gene and MYC oncogene in pathogenesis of human hepatocellular carcinoma: Potential prospects for combined targeted therapeutics (Review). Int J Oncol 41: 393-406, 2012.

44. Jeffers JR, Parganas E, Lee Y, et al: Puma is an essential mediator of p53-dependent and -independent apoptotic pathways. Cancer Cell 4: 321-328, 2003.

45. Zhang C, Zhang J, Zhang A, et al: PUMA is a novel target of miR-221/222 in human epithelial cancers. Int J Oncol 37: $1621-1626,2010$

46. Zhang CZ, Zhang JX, Zhang AL, et al: MiR-221 and miR-222 target PUMA to induce cell survival in glioblastoma. Mol Cancer 9: 229, 2010

47. Kim I, Moon SO, Koh KN, et al: Molecular cloning, expression, and characterization of angiopoietin-related protein. Angiopoietin-related protein induces endothelial cell sprouting. J Biol Chem 274: 26523-26528, 1999.

48. Kikuchi R, Tsuda H, Kozaki K, et al: Frequent inactivation of a putative tumor suppressor, angiopoietin-like protein 2 , in ovarian cancer. Cancer Res 68: 5067-5075, 2008.

49. Feng SY, Dong CG, Wu WK, Wang XJ, Qiao J and Shao JF: Lentiviral expression of anti-microRNAs targeting miR-27a inhibits proliferation and invasiveness of U87 glioma cells. Mol Med Rep 6: 275-281, 2012.

50. Park JK, Kogure T, Nuovo GJ, et al: miR-221 silencing blocks hepatocellular carcinoma and promotes survival. Cancer Res 71: 7608-7616, 2011 